\title{
LA TESIS DE LA IDENTIDAD ENTRE ESTADOS MENTALES Y ESTADOS FISICOQUIMICOS*
}

El presente trabajo tiene por objeto discutir la tesis que sostienen $U$. T. Place, J. J. C. Smart y D. M. Armstrong frente al problema mente-cuerpo.'

El propósito de Place y Smart es mostrar que la postulación de una identidad entre estados mentales y estados fisicoquímicos no puede desecharse $a$ priori sobre bases lógicas, y que no hay argumentos filosóficos válidos que muestren la imposibilidad de tal identidad. Ambos centran sus artículos en las sensaciones y los estados de conciencia, y su estrategia es responder objeciones basándose en la distinción significado-referencia de Frege y aduciendo analogias con identidades científicas. Argumentan que las objeciones en contra de la identidad solamente muestran que los significados y accesos cognoscitivos de los estados mentales son distintos a los de los estados fisicoquímicos, pero que una diferencia a nivel epistémico y semántico no implica una diferencia a nivel ontológico: ambas expresiones pueden referir a uno y el mismo estado, como es el caso de las expresiones "calor" y "movimiento acelerado de moléculas"."2

En defensa de la tesis de la identidad, Place y principalmente Smart han dicho lo siguiente:

1) A nivel epistémico y semántico, "sensación" y "proceso cerebral" tienen significados distintos y lógicamente independientes, diferentes lógicas de lenguaje, diferentes accesos cognoscitivos; por ello, cualquiera puede hablar correctamente sobre sensaciones sin saber nada de neurofisiología, de la misma manera que cualquiera puede hablar del calor sin conocer nada sobre la teoría molecular. Hay predicados que se aplican a los procesos cerebrales y que no tiene sentido aplicar a las sensaciones, y viceversa, como no tiene sentido decir que el movimiento de moléculas es seco o que el calor es rápido. Podemos imaginar que las sensaciones no son procesos cerebrales, como podemos imaginar que el calor no es movimiento de moléculas sino alguna otra cosa, ${ }^{3}$ pero,

* Agradezoo al Dr. Mark de Bretton Platts y al Dr. Enrique Villanueva sus excelentes comentarios e indicaciones en la elaboración de este trabajo, los cuales me permitieron eliminar errores y comprender mejor los problemas. No dudo que aún haya varios errores, pero éstos los debo solamente a mí misma.

1 La referencia principal son los articulos "EEs la conciencia un proceso cerebral?" de U. T. Place, "Sensaciones y procesos cerebrales" de J. J. C. Smart y "La naturaleza de la mente" de D. M. Armstrong. En Borst (ed.), The Mind-Brain Identity Theory, Macmillan, 1970.

2 J. J. C. Smart, op. cit., pp. 58 y 59.

3 Este último punto es cuestionable y parece traer serios problemas para la tesis de la identidad, como veremos más adelante. 
a nivel ontológico o metafísico, las sensaciones son idénticas a procesos cerebrales; asi como las expresiones "calor" y "movimiento acelerado de moléculas" tienen una y la misma referencia, el estado denotado por las expresiones "sensación" y "proceso cerebral tal y cual" es uno y el mismo -o al menos, se argumenta, esto es posible: la diferencia epistémica y semántica no garantiza diferencia ontológica. 1

2) Las llamadas "propiedades fenoménicas o psíquicas" pertenecen al nivel epistémico y semántico. No parece haber argumentos convincentes que demuestren la existencia de "objetos fenoménicos" con "propiedades fenoménicas": a nivel ontológico no hay tales cosas; sólo hay objetos físicos con propiedades físicas. ${ }^{5}$ Se dice, por ejemplo, que una imagen mental es amarilla o que un dolor es agudo, pero esto, arguye Smart, es una manera de hablar: ontologicamente no hay cosas tales como dolores o imágenes mentales; lo que hay es la experiencia de tener un dolor, o la experiencia de tener una imagen mental, y tales experiencias, ni amarillas ni agudas, son las que se propone identificar con procesos cerebrales. ${ }^{6}$

3) Smart dice que la incorregibilidad y la privacidad de las experiencias son una cuestión semántica de la lógica del lenguaje de reportes introspectivos, y no una propiedad ontológica de las sensaciones mismas. Esta afirmación no es clara y parece cuestionable; sin embargo, no me detendré en este punto.

Tanto Place como Smart argumentan que pasar del nivel epistémico y semántico - donde hay diferencia entre sensaciones y procesos cerebralesal nivel ontológico es cometer la falacia epistemológica o la fenomenológica; que el estado denotado por la palabra "sensación" puede ser un estado fisicoquímico con propiedades puramente fisicoquímicas.

Armstrong ve que para establecer la posibilidad de la tesis de la identidad no basta responder objeciones; el problema radica en mostrar que ni nuestros conceptos mentales involucran nada que implique un dualismo ni nuestro conocimiento actual de la presencia de estados mentales nos lleva, necesariamente, a aceptar una posición dualista. ${ }^{7}$

El trabajo se ha dividido en dos partes: la primera es un resumen del artículo de Armstrong; en la segunda se señalan algunos problemas para las tesis alli planteadas.

Hay muchos problemas por discutir involucrados en lo que aquí digo y sobre los cuales no hablo; pero, al presente, aún no tengo respuesta para ellos ni sé qué decir al respecto. Espero, en un futuro, poder responder a algunos

4 U. T. Place, op. cit., pp. 45-46; J. J. C. Smart, op. cit., pp. 57, 58, 59, 62, 63 (réplicas).

5 Parece sumamente dudoso que en el caso de sensaciones y experiencias conscientes podamos distinguir entre un nivel epistémico, de mera apariencia, y un nivel ontológico de realidad, en cuanto a propiedades - como lo hacemos en el caso de eventos del mundo externo, como el calor, por ejemplo. Este punto se comenta en la segunda parte.

6 U. T. Place, op. cit., pp. 48-51; J. J. C. Smart, op. cit., pp. 61 y 62.

7 He elegido el articulo de Armstrong para exponer la tesis de la identidad porque considero que es el más claro, además de que recoge los puntos importantes de los otros dos artículos exponiéndolos dentro de un mismo contexto: nuestro concepto de estado mental. 
de estos problemas, así como esclarecer varios puntos que ahora pueden resultar oscuros.

\section{Posición de Armstrong frente al problema mente-cuerpo}

1. Armstrong piensa que decir que "los hombres tienen mente es decir que tienen percepciones, sensaciones, emociones, creencias, pensamientos, propósitos y deseos". Así, preguntar qué es tener mente es preguntar qué es tener estados mentales y estar en un cierto estado mental es tener, por ejemplo, cierta percepción.

Nosotros tenemos el concepto de estado mental y, normalmente, sabemos aplicarlo correctamente en nuestro uso del lenguaje. ¿Cuál es nuestro concepto de estado mental? ¿Qué es estar en un cierto estado mental (tener una creencia, un deseo, etcétera)? Armstrong trata de dar respuesta a estas preguntas.

Tradicionalmente se ha pensado que el que los hombres tengan estados mentales implica un dualismo; que nuestro concepto de estado mental es incompatible con nuestro concepto de estado físico; que los estados mentales tienen propiedades que los estados físicos no tienen y viceversa; que la existencia de unos no implica la existencia de los otros. Que la mente es un "misterioso espectro interno" (del cual no tenemos un conocimiento que nos permita explicar ni predecir su comportamiento ni explicar su relación con el cuerpo) responsable de la conducta y algunos estados físicos del cuerpo.

Armstrong acepta una concepción fisicalista del hombre. Bajo este punto de vista el hombre no es más que un mecanismo fisicoquímico complejo: no hay nada en una persona, incluyendo todas sus características psicológicas o mentales, sobre o por encima de su cuerpo y sus características físicoquímicas. El propósito del artículo de Armstrong es ofrecer un análisis del concepto de estado mental que haga plausible esta opinión. Armstrong desea mostrar que el fisicalismo no es paradójico, sino completamente compatible con nuestros conceptos mentales ordinarios; que la identidad entre estados mentales y estados físicos no requiere alteración alguna en estos conceptos, ya que ellos no involucran la existencia de sustancias o propiedades no-físicas: tan sólo se aplican a condiciones causales relacionadas con cierto tipo de conducta.

2. Entre las teorias sobre los estados mentales se encuentra la conductista. Semánticamente, el conductismo postula la posibilidad de traducir los enunciados sobre estados mentales a enunciados sobre conducta manifiesta; es decir, afirma que el significado de expresiones mentales puede darse en términos de conducta manifiesta. Ontológicamente, postula (en una de sus versiones) que los estados mentales no son algo detrás de la conducta sino que son parte de la conducta misma. Identificar los estados mentales con la conducta corporal resulta muy atractivo para los filósofos que, como Armstrong, tienen una concepción fisicalista del hombre. Sin embargo, esta explicación presenta dificultades que Armstrong señala: la alegria, diría un conductista, no es un estado o modificación de alguna sustancia interna que de alguna manera causa o produce conducta alegre; la alegría es la conducta alegre misma. Pero 
un hombre puede estar muy alegre y no presentar ninguna conducta corporal al respecto; por tanto, los estados mentales y la conducta corporal no pueden ser lo mismo.

La respuesta más aceptable a esta objeción, según Armstrong, es la de introducir la noción de "disposición a comportarse" e identificar los estados mentales con disposiciones. Armstrong dice que, para los conductistas, tener una disposición no es estar en un estado particular, sino hallarse determinado - propenso a estar en un estado particular; que las disposiciones "no son algo", en el sentido de que no son estados reales de los objetos, sino que son tan sólo la posibilidad de estar en un estado real: conducta manifiesta.

La objeción de Armstrong a la noción de disposición que él mismo señala es que tal explicación de la presencia de estados mentales resulta inaceptable en el caso de la primera persona. Cuando yo siento un dolor y mi dolor no se manifiesta en conducta alguna, hay algo que está sucediendo en mi realmente. No es tan sólo que tenga una propensión a comportarme de cierta manera: hay algo real en mí que es o constituye mi sensación de dolor.

Armstrong dice que la explicación conductista de la presencia de estados mentales parece antinatural: cuando alguien siente un dolor y emite un quejido, parece antinatural decir que su sensación de dolor es su emisión del quejido; de manera natural más bien diríamos, según Armstrong, que su sensación de dolor es "la causa por la cual el hombre emitió el quejido, que el quejido es la expresión de su sensación de dolor" y que una cosa es la sensación de dolor misma (que es algo que sucede realmente), y otra, la expresión de tal sensación: que los enunciados sobre sensaciones refieren a algún tipo de estado interno del individuo de quien se predican.

3. Basándose en su crítica al conductismo, Armstrong dice que, como se ha pensado tradicionalmente, nuestro concepto de estado mental vincula la idea de que los estados mentales son estados internos reales (no meras posibilidades) y que son la causa de que las personas se comporten de cierta manera bajo determinadas circunstancias. Si esto es así, afirma Armstrong, entonces "nuestra noción de mente y de estado mental individual está lógicamente ligada a la conducta", como piensan los conductistas, sólo que ellos se equivocan al identificar ambas cosas.

Armstrong parece suponer que existen implicaciones entre enunciados mentales y enunciados sobre conducta; implicaciones que se siguen, en algún sentido, del significado de palabras mentales. Pero no porque los estados mentales sean idénticos a tal conducta o a una "disposición" a comportarse en tal forma, sino porque lo que entendemos por un estado mental es un cierto estado de la persona, que causa dicha conducta. Así, Armstrong pensaría que del enunciado " $\mathrm{X}$ tiene un dolor" se sigue el enunciado " $\mathrm{X}$ tiene un cierto estado que es apto para causarle tales y cuales conductas manifiestas".

Con base en lo anterior, Armstrong formula el concepto de estado mental en los siguientes términos: "Un estado mental es un estado de una persona apto para producir cierto tipo de conducta."

Es importante notar la similitud entre el análisis de Armstrong y el análisis conductista con "disposiciones". Un conductista diría: "tener un estado 
mental es tener una disposición a comportarse de cierta manera". Armstrong dice: "tener un estado actual apto para producir cierto tipo de conducta". En ambos casos el análisis asume que los enunciados mentales tienen conexiones lógicas (por su significado) con ciertos enunciados de conducta, sólo que Armstrong identifica al estado mental con un estado actual apto para causar la conducta en cuestión.

Armstrong parece sostener una relación inferencial entre los estados mentales y la conducta manifiesta: si lo que observamos directamente (en terceras personas) es la conducta manifiesta y ésta es distinta de los estados mentales, entonces los estados mentales no se observan directamente, sino que se infieren a partir de la conducta. Asi como de un evento observado inferimos una causa, de la conducta-tipo observada inferimos la presencia de estados mentales, ya que nuestro concepto de estado mental es el estado-causa de la conducta-tipo observada.

4. Al análisis de Armstrong se le opone una seria objeción, a saber, parece dejar fuera la conciencia y las experiencias propias. Si nuestro concepto de estado mental lo aplicamos sólo por inferencia, a partir de la conducta observada en terceras personas, entonces el análisis puede ser aceptable; pero cada uno de nosotros se adscribe directamente conceptos mentales, por medio de la conciencia de sus propias experiencias (por ello inferimos estados mentales en terceras personas al observar una conducta similar a la nuestra). ¿Podemos decir que lo que entendemos por conciencia es un estado interno de una persona apto para producir cierto tipo de conducta? Parece que no: un autómata - una persona no consciente - puede presentar el mismo tipo de conducta que una persona consciente.

Entre lo que entendemos por conciencia se encuentra el acceso directo a nuestros estados mentales propios. Si esto es así, debemos conocer la naturaleza de nuestros estados mentales propios, puesto que los experimentamos directamente. Mi concepto de dolor no es tan sólo "un estado interno apto para causar conducta dolorosa"; aun cuando esto sea verdadero, mi concepto de dolor incluye mi experiencia consciente de mi propia sensación, esa sensación específica que yo experimento y la manera como la experimento.

La respuesta de Armstrong a esta objeción es que su análisis puede ofrecer una explicación de la conciencia y de la presencia de experiencias propias. Su argumento es el siguiente: no podemos observar directamente los estados mentales de otros, pero cada uno de nosotros tiene la capacidad de observar directamente sus propios estados mentales y a esta capacidad es a lo que llamamos conciencia. Alguien que tiene conciencia es alguien que está informado de lo que sucede en su propia mente, alguien que "percibe" sus diferentes estados mentales. La objeción dice que el análisis propuesto no toma en cuenta la conciencia; pero si lo que entendemos por conciencia es un tipo de "percepción interna", ¿por qué no aplicarle un análisis similar al de la percepción externa?

Podemos hablar de la percepción externa como de estados internos aptos para producir cierto tipo de conducta selectiva hacia el medio ambiente. Si un niño no es capaz de distinguir entre objetos cuya única diferencia es el 
color, decimos que no percibe los colores, ya que no presenta ninguna conducta selectiva frente a los objetos; si el niño distinguiese los objetos diríamos que percibe los colores, ya que al tener los objetos enfrente algo ocurre dentro de él: un estado interno que es apto para producir la conducta selectiva que esperamos.

De la misma manera podemos concebir la conciencia como un estado interno dirigido hacia otros estados internos (no hacia el medio ambiente), el cual nos permite actuar de manera selectiva hacia nuestros estados mentales propios. Y, así como la vista nos informa de la presencia de luz sin determinar su naturaleza específica y nos da un conocimiento de la luz que no incluye sus propiedades esenciales, la conciencia nos informa de la presencia de estados mentales en nosotros mismos sin determinar su naturaleza ni sus propiedades esenciales.

Para Armstrong la conciencia no es sino un estado mental (de segundo orden) cuya función consiste en el escrutinio de nuestros estados mentales; un estado interno que nos da la capacidad de discriminar entre nuestros estados mentales y expresarlos conductualmente, si asi se desea. La conciencia nos informa que "algo ocurre dentro de nosotros", pero no șabemos qué sea ese "algo", en el sentido de que desconocemos su naturaleza.

5. Concebir los estados mentales como estados actuales aptos para producir cierto tipo de conducta no implica una concepción fisicalista de los estados mentales, pero la hace posible en la medida en que:

a) Los estados mentales son estados que ocurren actualmente; por tanto, es posible tratar de determinar su naturaleza, en tanto son estados aptos para producir cierto tipo de conducta (o informarnos sobre nuestros estados mentales propios en el caso de la conciencia).

b) Si la posibilidad de determinar la naturaleza de los estados mentales queda abierta (si la conciencia no nos informa sobre su naturaleza y nuestros conceptos no especifican nada al respecto, quizá otro medio de observación pueda hacerlo), entonces es posible que tal naturaleza quede determinada como puramente física: un estado físico puede ser un estado interno apto para producir cierto tipo de conducta.

Lo que Armstrong sostiene es que no hay nada más implicado o vinculado por nuestros conceptos mentales que lo que señala su análisis y, si éste es correcto, no hay nada en nuestros conceptos mentales que elimine la posibilidad de identificar los estados mentales con estados físicos del cuerpo.

El análisis conceptual es neutro respecto a la naturaleza intrínseca de los estados mentales; tan sólo dice que son intermediarios en una cadena causal entre estímulo físico y conducta manifiesta. Así, si la cadena causal puede explicarse completamente en términos físicos, entonces los estados mentales se identificarán con los estados físicos intermediarios, sin que para su explicación se presupongan atributos no-físicos adicionales.

Podemos esquematizar la propuesta de Armstrong como sigue: 
Cadena causal de un proceso físico:

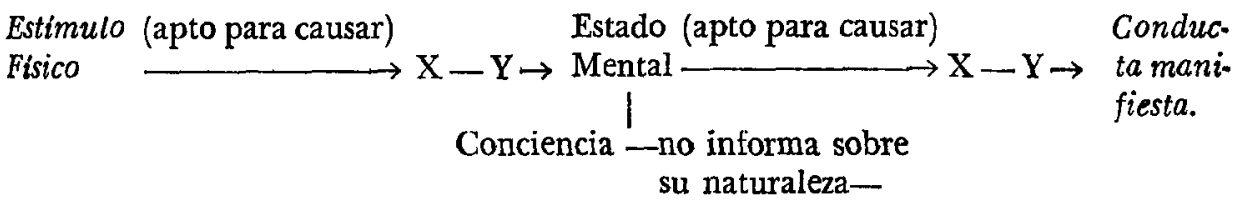

(mecanismo informador que da la capacidad de discriminar entre estados mentales).

6. El identificar los estados mentales con estados ffsicos del cuerpo depende de que la naturaleza o composición de aquello que entendemos por un estado mental quede determinada como función fisicoquímica del cuerpo. Pero cómo determinar esta naturaleza? La respuesta de. Armstrong es que dicha tarea corresponde exclusivamente a la ciencia.

La tesis de la identidad se propone como una hipótesis científica plausible; la posibilidad del fisicalismo la establece el análisis conceptual, pero su verdad quedará establecida por los descubrimientos e hipótesis científicos. Armstrong piensa que si la ciencia logra explicar toda la conducta del hombre en términos puramente fisicoquímicos, deberemos aceptar que no hay nada en el hombre, incluyendo los estados mentales, que no sea de naturaleza fisicoquimica. Si un estado mental es el estado-causa (apto para causar cierto tipo de conducta) y la ciencia descubre que la causa de tal conducta es el funcionamiento fisicoquimico del sistema nervioso central, entonces podremos identificar los estados mentales con estados fisicoquímicos de dicho sistema y la conciencia con el mecanismo auto-informador (auto-escudriñador) del mismo.

El argumento de Armstrong a favor de la autoridad científica se basa en el "hecho" de que sólo como resultado de investigaciones cientificas se logra un consenso intelectual sobre lo que es el caso y que, a excepción de las matemáticas y las ciencias naturales, no hay nada cierto o aceptado como verdadero en ninguna otra disciplina estudiada.

Armstrong no niega que las verdades y certezas de la ciencia sean relativas y falibles, pero argumenta que no tenemos ninguna guía mejor que las verdades y certezas aceptadas en el presente y, si la ciencia nos dice que la naturaleza del hombre es puramente fisicoquímica, debemos aceptar esto como cierto . (en el presente), aun cuando puede ser que se equivoque. También concede que quizá la ciencia no pueda ayudarnos a decidir algunas cuestiones, pero arguye que hasta el momento no hay otra disciplina capaz de decidir cuestiones y que, por tanto, debemos aceptar toda la evidencia que la ciencia pueda darnos.

7. El tipo de identidad que se propone tiene las siguientes caracteristicas:

a) Es una identidad ontológica. Se acepta que epistémica y semánticamente "estado mental" es diferente de "estado fisicoquímico": los significados de expresiones sobre estados mentales y de expresiones sobre estados fisicoquímicos son diferentes; los accesos cognoscitivos 'conciencia' y 'conducta observada' 
son distintos e independientes del acceso cognoscitivo científico. Y se propone que el estado conocido y denotado en todos los casos es uno y el mismo ontológicamente. 8

Esta propuesta se apoya en la distinción fregeana entre significado y referencia: expresiones con significados distintos y logicamente independientes no implican una referencia diferente; diferentes accesos cognoscitivos, diferentes observaciones y verificaciones no implican diferentes objetos o eventos conocidos, observados y verificados.

b) Es una identidad contingente. Es lógicamente posible que expresiones con diferentes significados tengan diferentes referencias, pero también es lógicamente posible que tengan una y la misma referencia; el que ocurra uno o lo otro es una cuestión de hecho. Por ejemplo, "agua" y "moléculas $\mathrm{de}_{\mathrm{H}} \mathrm{O}$ " podrían haber referido a dos cosas diversas, pero de hecho refieren a uno y el mismo suceso. La verdad de la identidad no se establece a priori por necesidad lógica, sino a posteriori, como un resultado de descubrimientos científicos. ${ }^{9}$ (Los teóricos de la identidad parecen unir la noción de contingencia a la noción de conocimiento a posteriori; sin embargo, éstas son dos nociones distintas e independientes, como veremos en la segunda parte, problema 3.)

Lo que se quiere decir con que el enunciado de identidad " $A$ es $B$ " sea contingente no es que sea contingente que $A$ y $B$ ocurran juntos -esto sería suponer falso el enunciado de principio, pues si $A$ y $B$ refieren a un mismo estado $X$, entonces, si ocurre $X$, necesariamente ocurre $X$-, sino que es contingente que las expresiones $A$ y $B$ refieran a uno y y el mismo estado $X .^{10}$ Pero si los referentes de ambas expresiones son uno y el mismo, entonces, sí es verdad que ocurre el estado que llamamos $A$, es necesariamente verdadero que ocurre el estado que denotamos con la expresión $B$ y viceversa. Si las expresiones tienen diferentes significados, no todo lo que es verdadero de una es verdadero de la otra; pero si tienen idéntica referencia, todo lo que es verdadero del estado reportado en términos mentales debe ser verdadero del estado reportado en términos físicos.

c) Es una identidad asimétrica, en el sentido en que se propone que todo estado mental es, ontológicamente, un estado físico, y no que los estados físicos sean, ontológicamente, estados mentales.

Aun cuando fuese verdadero decir que algunos estados físicos del sistema nervioso central son estados internos de las personas aptos para... (o estados mentales), el término de la izquierda es el que especifica la naturaleza ontológica del estado reportado vagamente por el término de la derecha. ${ }^{11}$

8 "...la tesis no afirma que los enunciados sobre sensaciones puedan ser traducidos a enunciados sobre procesos cerebrales. Ni tampoco afirma que la lógica de un enunciado sobre sensaciones sea la misma que aquella de un enunciado sobre procesos cerebrales. Todo lo que afirma es que, en tanto que un enunciado de sensaciones es un reporte de algo, ese algo es, de hecho, un proceso cerebral". J. J. C. Smart, op. cit., p. 56.

- " $\mathrm{La}$ estrella matutina y la estrella vespertina son una y la misma cosa' es una proposición contingente." J. J. C. Smart, op. cit., p. 59.

10 J. J. C. Smart, op. cit., p. 59 (réplica).

11 "Pero si estamos convencidos, sobre fundamentos científicos generales, que una explicación puramente f́sica del hombre es probablemente la verdadera, entonces parece no ha- 
La asimetría de la identidad puede explicarse por la distinción significadoreferencia: ambas expresiones tienen una misma referencia, pero el significado de la expresión "estado mental" es totalmente neutral respecto a la naturaleza del fenómeno, mientras que el significado de la expresión en términos físicos proporciona información sobre dicha naturaleza, determinando con ello el status ontológico del estado denotado por dichas expresiones.

d) Es una identidad tipo. Armstrong dice que podemos ver la percepción (externa) como estados internos; con ello se refiere a la percepción en general, es decir, como una clase de eventos o sucesos, a saber, la clase de eventos de la forma " $X$ percibe $Y$ en $T$ ". Se habla de un estado mental como de la clase o tipo de varios eventos particulares: un tipo de conducta, por ejemplo, la conducta dolorosa, es causada por un tipo de estado mental, la sensación de dolor, y la naturaleza de ese estado-causa es física: el tipo de estado $X$ del sistema nervioso central. ${ }^{12}$

La identidad no se postula: "el estado mental $X$ de $Y$ en $T$ es el estado $Z$ del sistema nervioso central de $Y$ en $T$ " sino: "la clase de estado mental $X$ es la clase de estado $Z$ del sistema nervioso central".

No tan sólo se propone identificar mi dolor de cabeza particular, que ocurre en el tiempo $T$, con el estado particular de mi sistema nervioso central en $T$; se postula que la clase de dolores $D$ es idéntica a la clase de los estados $C$ del sistema nervioso central. Y una razón por la cual la identidad se postula como una identidad tipo es la de hacer posible que quede establecida por la ciencia conforme a leyes.

\section{Algunos problemas para Armstrong}

1. Armstrong dice que lo que entendemos por un estado mental es "un estado interno de una persona apto para producir cierto tipo de conducta", y, por tanto, que decir que alguien se encuentra en un estado mental es decir que se encuentra en un estado interno apto para producir $Y$ tipo de conducta. Sin embargo, esta afirmación presenta algunos problemas:

a) ¿Sólo las personas pueden tener estados mentales? La atribución de un estado mental ¿conlleva la aplicación de nuestro concepto de persona (cualquiera que éste sea)?

Parece que si aplicamos nuestro concepto de persona a un organismo, le

ber ningún obstáculo para identificar estos estados internos con estados puramente físicos del sistema nervioso central." D. M. Armstrong, op. cit., p. 79.

“...es sólo en ciencia donde los hombres (...) alcanzan un acuerdo sustancial sobre lo que es el caso". D. M. Armstrong, op. cit., p. 68.

“...los procesos reportados en enunciados de sensaciones son de hecho procesos en' el cerebro". J. J. C. Smart, op. cit., p. 56.

12 "En general, podemos pensar en la percepción como estados o eventos internos aptos para la producción de cierto tipo de conducta selectiva hacia nuestro medio ambiente (...) alguien que está consciente de sus pensamientos o sus emociones es alguien que tiene la capacidad de hacer discriminaciones entre sus diferentes estados mentales." D. M. Armstrong, op. cit., p. 78. 
atribuimos la capacidad de tener estados mentales; pero creo que estamos dispuestos a atribuir estados mentales sin aplicar nuestro concepto de persona: decimos que un perro percibe, siente hambre, se enoja; $y$, sin embargo, no decimos que un perro sea una persona.

Parece que los estados mentales no son estados internos de las personas exclusivamente; al menos, comúnmente no siempre que aplicamos conceptos mentales aplicamos el concepto de persona. Si esto es correcto Armstrong estaría capturando en su análisis sólo un subconjunto de los estados mentales y no el conjunto completo, por así decirlo.

b) ¿Qué tipo de conexión lógica hay entre la conducta y nuestra noción de estado mental? Si lo que entendemos por estado mental es un estado interno apto para producir cierto tipo de conducta, entonces debería haber algún tipo de equivalencia entre ambos; al menos, tal requerimiento parece natural. Pero cacaso todo aquel que presenta un cierto tipo de conducta (o que tiene estados internos aptos para producir cierto tipo de conducta) se encuentra en un estado mental?

Supongamos un robot que presentara un tipo de conducta similar al humano; que al recibir un daño en su cuerpo realizara movimientos corporales del tipo de la conducta dolorosa de los humanos. Obviamente tales movimientos serían causados por un estado interno apto para producirlos (procesos fisicoquímicos quizá del tipo de los que ocurren en los humanos cuando presentan conducta dolorosa), pero cbastaría esto para decir que el robot siente dolor al recibir un daño en su cuerpo? Podriamos afirmar que el robot no siente dolor, que simplemente está diseñado y programado para responder ante determinadas circunstancias con una conducta similar a la conducta dolorosa de los humanos.

Si esto es posible, el análisis de nuestros conceptos mentales propuesto por Armstrong resultaria insuficiente: observar en un sujeto cierto tipo de conducta y la presencia de estados internos aptos para producir tal conducta, no parece suficiente para que le adscribamos estados mentales. Nosotros asumimos que el sujeto en cuestión experimenta algo; si hay alguna razón para pensar que el robot no experimenta nada al recibir daño en su cuerpo, no vamos a decir que siente dolor, aun cuando se encuentre en un estado interno apto para producir el tipo de conducta que los humanos presentan cuando decimos que sienten dolor. (Esto nos lleva a otro problema, que trataré en el punto 2).

En el ejemplo anterior, los enunciados sobre conducta y sobre estados internos aptos para producir tal conducta son verdaderos, mientras que los enunciados sobre estados mentales son falsos -o podrían serlo: quizá sea posible construir un robot que experimente algo, pero ello no se sigue de la verdad de los enunciados sobre conducta y estados internos: podemos decir sin contradicción que el robot no experimenta nada cuando recibe daño en su cuerpo, aun cuando presenta un tipo de conducta similar a la conducta dolorosa de los humanos.

¿Es posible imaginar casos donde los enunciados sobre estados mentales sean verdaderos y los enunciados sobre estados aptos para producir cierto tipo 
de conducta sean falsos? Podemos pensar en un organismo que siente dolor pero se encuentra físicamente imposibilitado para presentar ningún tipo de conducta dolorosa, o bien tiene la creencia de que cada vez que siente dolor debe suprimir cualquier manifestación conductual de su sensación. En defensa de Armstrong podría alegarse que la sensación de dolor es apta para... en el sentido de que si todas las circunstancias son propicias, el estado se hace eficaz en su función causal, y que en los casos que hemos imaginado sucede simplemente que las circunstancias no son propicias, no que no haya un estado interno apto para... Pero entonces podríamos preguntar cuáles son las circunstancias propicias, pregunta que presenta algunos problemas.

Suponiendo que aceptamos el esquema de análisis, ¿cómo vamos a especificar el análisis para un estado mental determinado y un tipo de conducta tal y cual?

Dado el estado mental, ¿cómo especificamos el tipo de conducta que es apto para producir? El enunciado " $X$ siente comezón" se analizaría: " $X$ tiene un estado interno que es apto para producir que $X$ se rasque"; la pregunta es ecuándo es apta la sensación de comezón para causar la conducta de rascarse?

Podría haber alguien a quien sentir comezón le causara apretarse los lóbulos de las orejas porque creyese que la comezón se quita con ello, y deseara suprimir su sensación; o bien alguien a quien la sensación de comezón le causara conducta alegre porque creyese que tal sensación es signo de buena suerte: le diera alegría tener buena suerte y no deseara suprimir su sensación.

Parece que la sensación de comezón es apta para causar la conducta de rascarse si, por un lado, las circunstancias ambientales son propicias y el sujeto tiene la posibilidad física de hacerlo; por otro, si ese alguien cree que rascarse suprimirá la sensación, desea suprimir la sensación...

Así pues parece que del enunciado " $X$ siente comezón" no podemos inferir el enunciado " $X$ tiene un estado interno apto para causar que $X$ se rasque", a menos que atribuyamos a $X$ ciertos estados mentales adicionales. Parece que no es sólo la conducta tipo observada lo que nos lleva a atribuir un estado mental tipo, sino que también las circunstancias especificas juegan un papel muy importante en la atribución de tal o cual estado mental; cada atribución de un estado mental parece estar vinculada a una serie de circunstancias, entre las cuales se encuentran otros estados mentales. (Si vamos al teatro y uno de los actores presenta conducta dolorosa, pensaremos quizá que tiene un estado interno apto para producir tal conducta; sin embargo, lo más probable es que no le atribuyamos sensación de dolor, sino ciertas intenciones y creencias.)

El problema es que el análisis debe darse en términos de causas de conducta manifiesta sin incluir estados mentales, pero ¿cómo deshacernos de los enunciados mentales? Parece que cada vez que intentemos sustituir un enunciado mental por su análisis correspondiente tendremos exactamente el mismo problema. ¿Dónde se detendrá el análisis?

Dado el tipo de conducta manifiesta, ccómo determinamos el estado mental que la causó? Esto no parece estar determinado; por el contrario, hay va- 
rias opciones distintas para explicar un mismo hecho de conducta. Si un niño llora en la comida, se queja y dice que le duele el estómago, podemos decir, para explicar el hecho observado, que el niño siente dolor y que esta sensación es la causa de su conducta, pero también podemos atribuir al niño el deseo de no comer y la creencia de que fingir dolor evitará que coma. ¿Cómo vamos a decidir cuál explicación es la correcta, qué estado mental causa este tipo de conducta?

Según parece, diferentes enunciados sobre estados mentales podrían tener uno y el mismo análisis: "el estado interno apto para causar tal y cual con-. ducta". Pero lo que entendemos por dolor no es lo mismo que entendemos por tener ciertas creencias y deseos; así, pues, en el análisis de Armstrong parece faltar algo que especifique la diferencia.

c) Armstrong parece asumir que sobre la base de la conducta observada en terceras personas inferimos una causa de tal conducta: un estado "mental" apto para producirla (estado que puede ser físico). Esta propuesta resulta cuestionable porque, según parece, no hacemos inferencia alguna cuando atribuimos dolor a alguien cotidianamente; tales atribuciones parecen ser más bien irreflexivas.

Otra alternativa sería decir que efectivamente ocurren dos eventos distintos - la sensación de dolor de $X$ y la conducta dolorosa que $X$ presenta-, pero que no inferimos la presencia del primero sobre la base de haber observado el segundo, sino que en la conducta dolorosa de $X$ "vemos" directamente la sensación de dolor de $X$; al observar en la conducta de $X$ la expresión del dolor atribuimos dỉrectamente una sensación de dolor a $X$.

Esta propuesta parece señalar que la aplicación de conceptos mentales está vinculada a la conducta observable, pero no porque a partir de la conducta infiramos una causa - la presencia de un estado mental-, sino porque la conducta observable es nuestro patrón regulador del uso, aplicación y obtención o aprendizaje de tales conceptos. Si esto es correcto, entonces Armstrong se equivoca al pensar que la atribución de estados mentales está vinculada a la conducta de tal manera que al observar cierto tipo de conducta inferimos estados mentales como su causa; lo que realmente sucederia es que cierto tipo de conducta regula directamente nuestra aplicación de conceptos mentales sin necesidad de inferir causas.

2. Para Armstrong, la conciencia es un estado interno que da la capacidad de discriminar entre estados internos aptos para producir cierto tipo de conducta; tener conciencia es estar informado o "percibir" el estado interno que ocurre en nosotros. Pero parece que esta explicación de la conciencia no captura todo lo que entendemos por conciencia.

En el ejemplo del robot dejamos pendiente un punto, a saber, las experiencias. Según Armstrong, podemos reducir la percepción (externa) a estados internos aptos para producir cierto tipo de conducta selectiva para con el medio ambiente; así, tener una experiencia perceptual consciente sería poder discriminar el estado interno que es la causa de que uno sea capaz de presentar cierta conducta selectiva frente a ciertas circunstancias ambientales. Pero una máquina puede presentar conducta selectiva para con el medio ambiente y 
estar informada del estado interno que se produce en ella cada vez que esto sucede, mas no por ello decimos que la máquina tiene experiencias perceptuales conscientes. Podemos adscribir experiencias perceptuales a los organismos sobre la base de su conducta (selectiva para con el medio ambiente), pero creemos que en cada caso estas experiencias tienen también un carácter subjetivo específico, un punto de vista particular. Pensamos que si un organismo tiene conciencia, entonces hay algo que es ser como ese organismo, algo que es sentir y experimentar como siente y experimenta el organismo. Parece que no podemos desligar lo que entendemos por "experiencia consciente" de lo que experimenta un organismo, de lo que es para un organismo tener una experiencia.

Una posición que me parece pertinente considerar es la que sostiene que la conciencia nos da un conocimiento de propiedades esenciales de nuestras experiencias propias; que es esencial de toda experiencia ser experimentada de cierta forma, desde un punto de vista particular. Armstrong no parece tomar en serio esta objeción, pero parece ser que llamamos "dolor" (por ejemplo) a una cierta experiencia consciente que padece un organismo, y que cuando atribuimos sensación de dolor suponemos la presencia en un organismo de una experiencia determinada, misma que nosotros experimentaríamos si fuésemos ese organismo. Podemos construir un robot con diferentes estados internos y entre ellos uno que le dé la capacidad de discriminar entre sus estados internos aptos para... Dicho robot estará informado del tipo de estado interno que existe en él cada vez que presenta un tipo de conducta similar, por ejemplo, a la conducta dolorosa de los humanos, y quizá podrá informarnos al respecto; sin embargo, es dudoso que estemos dispuestos a aplicar al robot nuestro concepto de conciencia: no dirfamos que tiene un estado interno que le da la capacidad de discriminar entre..., si asumimos que no hay nada que sea sentir como el robot, que si fuésemos el robot no experimentaríamos nada.

Plantearé de otro modo la cuestión a través de un cuento: un buen día llegan a la tierra seres de otro planeta que tienen un aparato perceptual totalmente distinto al nuestro y cuya misión consiste en saber qué es percibir como un ser humano, qué es tener las experiencias perceptuales que tiene un humano. Los extraterrestres podrían adscribirnos algún tipo de percepción sobre la base de nuestra conducta y estructura física; pero sabemos que se equivocarian al afirmar que solamente puede atribuírsenos un tipo general de percepción, es decir, que no hay nada específico que sea percibir como nosotros; porque sabemos que nuestras experiencias perceptuales (y en general todas nuestras experiencias conscientes) poseen un carácter subjetivo sumamente específico, mismo que el análisis de Armstrong parece no capturar.

De hecho, los extraterrestres de nuestro cuento parecen interesados en conocer el carácter subjetivo de las experiencias perceptuales humanas, el punto de vista de los humanos. No quieren saber qué serfa para ellos comportarse como se comporta un humano, o encontrarse en las situaciones en las que se encuentra un humano, sino, más bien, qué sería percibir como un humano, qué es, para un humano, percibir como un humano, qué es ser como un ser 
humano. ¿Cómo pueden llegar a saber tal cosa? Sus aparatos perceptuales son totalmente distintos a los nuestros y no parece haber razón alguna para suponer que nuestras experiencias perceptuales sean, subjetivamente, como nada de lo que ellos puedan experimentar o imaginar. ¿Cómo podrían saber qué es para un ser humano percibir la luz por medio de la vista si carecen de este sentido; cómo podrían imaginar qué experimenta un humano cuando percibe la luz?

Las experiencias conscientes parecen implicar un punto de vista particular - lo que es para el organismo experimentador tener una cierta experienciay parece que, siempre que tratamos de concebir las experiencias de otro organismo distinto a nosotros, "transportamos" nuestro punto de vista: concebimos o imaginamos lo que sería para nosotros encontrarnos en una situación similar. Entre más semejante a nosotros sea el organismo en cuestión, más similar al nuestro parecerá ser su punto de vista; entre menos se nos asemeje, menos posible parecerá concebir o imaginar qué experimenta, pues, para saber qué es ser como un $X$, parece requerirse que uno adopte el punto de vista de un $X$. Si uno puede adoptarlo parcial o aproximadamente, entonces su idea de qué es ser como un $X$ será parcial o aproximada; si no puede adoptarlo, ni siquiera parcialmente, entonces no tendrá ni siquiera una idea parcial de qué es ser como un $X$.

Si lo anterior es correcto, si las experiencias conscientes son accesibles - completamente comprensibles - para un punto de vista particular (nótese que no se dice para un individuo: varios individuos, suficientemente similares entre sí, pueden tomar un punto de vista: un ser humano cualquiera sabe lo que es ser como un ser humano, cualquier humano que puede ver sabe lo que es percibir la luz como un humano), entonces parece dificil entender cómo es que el verdadero carácter de las experiencias pueda especificarse por medio de los procesos fisicoquímicos de los organismos. Tales procesos pertenecen a la clase de eventos que pueden ser comprendidos por diferentes puntos de vista - por organismos con diferentes sistemas perceptuales y diferentes estructuras - y no es fácil entender cómo es que la verdadera naturaleza de los estados subjetivos nos sea revelada por medio de estados objetivos.

Generalmente, cuando tratamos de establecer la "naturaleza real" de las cosas, las describimos en términos de sus propiedades detectables por cosas distintas de los sentidos humanos; trazamos una división entre cómo se nos aparecen y cómo son en realidad -es decir, independientemente de las im. presiones que producen en nuestros sentidos o en los de otros organismos. Es posible realizar un movimiento de la apariencia a la realidad en varios casos (identidades cientificas) porque nuestros conceptos sobre el mundo externo refieren a cosas que no están conectadas con un punto de vista particular: no es necesario que el objeto o evento denotado esté unido a ese punto de vista que lo "percibe" inicialmente de cierta manera particular. En la búsqueda de objetividad abandonamos los puntos de vista particulares en favor de una descripción accesible a diferentes puntos de vista: quizá los extraterrestres puedan entender el evento físico del mundo externo al que llamamos luz, en términos de su descripción como fotones en movimiento ondulatorio (para 
ello no se requiere que entiendan las formas fenomenológicas en las que el evento en cuestión aparece a los sentidos humanos), pero estarfan conociendo el mismo evento: de esto no parece haber duda.

En el caso de las experiencias, la conexión con un punto de vista particular parece esencial; la idea de distinguir entre apariencia y realidad parece inoperante. ¿Tiene sentido preguntar cómo son realmente mis experiencias, en oposición con, o a diferencia de, cómo se me aparecen? Resulta difícil entender cómo podremos captar la naturaleza real de una experiencia abandonando el punto de vista particular del sujeto experimentador: ¿podemos, por medio de un estado objetivo, captar la naturaleza real de un estado subjetivo? Parece raro suponer que los extraterrestres de nuestra historia comprendan la naturaleza real de las experiencias humanas bajo una descripción fisicoquímica -accesible a ellos- y que no sean capaces de imaginar, ni siquiera aproximadamente, qué sería ser como un humano. Si dejan de lado la particularidad de nuestro punto de vista humano (como en el caso de los eventos del mundo externo), ¿qué estarán comprendiendo de las experiencias humanas conscientes?

Si el carácter subjetivo de las experiencias es completamente comprensible solamente para un punto de vista, entonces parecería que todo movimiento hacia una mayor objetividad en ellas, en la medida en que conllevaría menor adherencia a un punto de vista especifico, nos alejaría de su naturaleza real - de su comprensión total — en vez de acercarnos a ella.

No podemos entender la hipótesis de que la naturaleza de las experiencias puede especificarse en una descripción física, sin antes entender la idea de que las experiencias tienen una naturaleza objetiva; necesitamos entender cómo o en qué sentido un estado mental esencialmente conectado a un punto de vista particular -es decir, esencialmente subjetivo - puede tener un carácter objetivo. ¿Cómo podrá capturarse la subjetividad característica de las experiencias, el punto de vista particular del sujeto experimentador, identificándolas con procesos fisicoquímicos que parecen abandonar los puntos de vista particulares?

Parecería que, si los estados mentales son procesos físicos, entonces hay necesariamente algo que es como padecer ciertos procesos f́sicos; es decir, sería necesariamente verdadero de un cierto proceso físico el sentirse de una cierta manera, tener un cierto carácter subjetivo. Aquí no sucedería, como en el caso de los eventos del mundo externo, que uno y el mismo evento físico .impresionara de manera distinta los sentidos de dos organismos diferentes. ${ }^{13}$ Este punto nos lleva a otro problema.

3. En contra de la identidad entre mente y cuerpo se ha argumentado que no es posible concebir que siento un dolor y que no experimento la sensación especifica que ocurre en mí cuando digo que siento un dolor - de lo cual parece seguirse que mis experiencias conscientes propias son propiedades esenciales de lo que yo llamo tal o cual sensación. Sin embargo, es posible imaginar, por ejemplo, que siento un dolor y no ocurre en mi un proceso

13 En relación con este problema cf. Thomas Nagel, "What is it like to be a bat?", en Thomas Nagel, Mortal Questions, Cambridge University Press, U. S. A., 1979. 
cerebral $X$ (o un estado $X$ del sistema nervioso central); pero si la naturaleza de lo que entendemos por un estado mental tal y cual es el estado $X$ del sistema nervioso central, ésta debía ser una propiedad esencial del estado en cuestión.

El mismo argumento puede tener otra versión: puedo concebir que mi cuerpo haga todo lo que hace ahora, externa e internamente, con todas las causas físicas de su conducta compleja, sin que ocurra ninguno de los estados mentales que estoy experimentando ahora, ni ningún otro. Si esto es realmente concebible, entonces los estados mentales deben ser algo distinto de los estados físicos del cuerpo.

La respuesta de los teóricos de la identidad a este tipo de argumento es que nuestro concepto de estado mental es totalmente neutral al respecto: no especifica nada sobre la naturaleza de lo que llamamos "estados mentales" $\mathrm{y}$, por ello, es lógicamente posible imaginar que tal naturaleza no es física. Pero lo que se argumenta no es que los estados mentales, debido a todo lo que sabemos sobre ellos, puedan tener una naturaleza distinta a la física, sino que debido a que podemos concebir la existencia de estados mentales sin la presencia de estados del sistema nervioso central (o de cualquier otro estado físico del cuerpo), los estados mentales deben ser algo distinto de los estados físicos del cuerpo. Este argumento se basa en que si $X$ es idéntico a $Y$ -donde $X$ y $Y$ designan propiedades esenciales de un evento-, entonces no es concebible que ocurra $X$ y no ocurra $Y$ o viceversa.

En el análisis causal de Armstrong (suponiendo que la ciencia explique todo el proceso en términos puramente fisicoquímicos) la presencia de la causa física de la conducta humana debiera implicar la presencia de estados mentales en el hombre: así, pues, si Armstrong está en lo cierto (o si la tesis de la identidad es plausible) el objetante se equivoca (tiene ilusiones) al creer que puede concebir tal cosa.

La tesis de la identidad se postula como un caso de identidad contingente; con ella se pretende explicar, entre otras cosas, por qué podemos imaginar que los estados mentales no son estados físicos del cuerpo. Smart explica por qué es lógicamente posible imaginar que la tesis de la identidad es falsa: expresiones con significados distintos y lógicamente independientes pueden tener, lógicamente, diferente denotación, en cuyo caso la presencia del evento o estado denotado por una no implica la presencia del evento o estado denotado por la otra; pero también es lógicamente posible que tengan una misma denotación, y en este caso la presencia del evento denotado por una implica la presencia del evento denotado por la otra, aun cuando sea posible imaginar que las cosas podrían haber sido de otro modo. (Esto es como decir que es contingente -no necesario - que necesariamente ocurran juntos los eventos.) Sin embargo, en este sentido, no conocemos ningún caso de "identidad contingente". La supuesta analogía con identidades científicas falla en este punto: por ejemplo, no es posible imaginar que el evento del mundo externo al que denotamos con la palabra "calor" no sea el evento del mundo externo que describimos en términos de moléculas en movimiento. Podemos imaginar que tal evento no nos produce la sensación de calor que, normalmente, nos produce, 
ya que el producirnos calor es una propiedad contingente del evento; podemos imaginar que existen seres construidos de tal modo que el evento que nosotros describimos como movimiento acelerado de moléculas les produce lo que nosotros entendemos como sensación de frio, o de cosquilleo, etcétera, pero no podemos imaginar, sin alteraciones en nuestro lenguaje, que el evento denotado por una de las expresiones _ "calor" o "movimiento acelerado de moléculas"- no sea el mismo que el evento denotado por la otra. Es necesario que el calor y el movimiento de moléculas ocurran juntos necesariamente, dado que ambas expresiones refieren necesariamente al mismo objeto: conservando igual el lenguaje no podemos imaginar que se refieran a dos cosas distintas.

En el caso del dolor y el estado $X$ del sistema nervioso central, parecemos seleccionar el estado mental 'dolor' por una propiedad esencial del estado, a saber, la de producirnos sensación de dolor; y lo que se argumenta es que podemos imaginar que el estado interno denotado por la palabra "dolor" no es el estado interno que describimos en términos del proceso fisicoquímico $X$ del sistema nervioso central. Es obvio que en este caso no es tan sencillo argüir solamente que lo que imaginamos es que el estado interno en cuestión no nos produce sensación de dolor porque la experiencia de dolor es el dolor mismo; parece que la distinción apariencia-realidad no se aplica en estos casos.

El problema real es si uno puede concebir tal cosa realmente, o si creer que esto es concebible es un error. Lo que debe mostrarse para rechazar el argumento es, o bien que cuando tratamos de concebir la presencia de estados mentales sin nuestros cuerpos, o viceversa, no tenemos éxito (que concebimos alguna otra cosa que no son nuestros estados mentales o nuestro cuerpo mismos), o bien que la "experiencia consciente dolorosa" que $X$ experimenta cuando se encuentra en el estado de dolor no es una propiedad esencial del dolor. ${ }^{14}$

4. Armstrong propone una identidad tipo, pero una identidad tipo entre estados mentales y estados fisicoquímicos trae serios problemas. Aceptarla nos llevaría a decir, por ejemplo, que el estado mental 'creencia' es el estado fisicoquímico $X$ del sistema nervioso central y que el estado mental 'sensación de dolor' es el estado fisicoquimico $Y$ del sistema nervioso central. Pero ¿podemos aceptar que lo que es tener una creencia o sentir un dolor depende de la naturaleza física (del estado fisicoquímico) de los seres humanos? ¿Podemos establecer que es físicamente necesario que un organismo tenga una creencia o sienta un dolor si y sólo si se encuentra en un estado fisicoquímico $X \circ Y$ ? ¿Podemos decir que es físicamente necesario que un organismo tenga un sistema nervioso central para que sienta dolor?

Supongamos que descubrimos la existencia de criaturas con una apariencia muy similar a la nuestra, que presentan idéntica conducta que la nuestra y que, según todos los indicios, parecen tener creencias, sentir dolores, etcétera, tanto como parecen tenerlos otras personas hoy en dia, solamente que sus procesos fisicoquímicos son totalmente distintos a los nuestros y no tienen

14 En relación con este problema cf. Saul Kripke, "Identidad y necesidad", Cuadernos de Critica № 7 . 
sistema nervioso central ni nada que se le parezca. Si tomamos en serio la tesis de la identidad nos veremos llevados a decir que estas criaturas no tienen estados mentales, pero esta afirmación parece inaceptable ya que: a) identificamos los estados mentales sobre la base de que son estados internos aptos para producir cierto tipo de conducta (según propone Armstrong); b) parece físicamente posible que un organismo presente cierto tipo de conducta -conducta dolorosa, por ejemplo- aunque no se encuentre en el estado fisicoquímico $Y$ sino en un estado fisicoquímico $Z$ (¿qué justifica la imposibilidad física en un caso tal?); ${ }^{15} c$ ) intuitivamente, atribuiriamos creencias y sensaciones de dolor a criaturas con idéntica apariencia y conducta que la humana. Nuestros criterios de atribución de estados mentales son a priori - en el sentído de que cualquiera que conoce y entiende el lenguaje sabe, por ese hecho, aplicar predicados mentales - e independientes de los descubrimientos cientificos sobre la composición fisicoquímica de los organismos a los que se los atribuimos; y cambiar nuestros criterios ordinarios de atribución de estados mentales sería cambiar en gran medida nuestros conceptos mentales ordinarios, en cuyo caso podría argumentarse que no se está identificando lo que llamamos "estado mental", sino alguna otra cosa más o menos similar (en sus relaciones causales), ya que nuestros conceptos mentales ordinarios son perfectamente aplicables a criaturas como las del ejemplo.

Parece más sensato identificar este evento mental particular de $X$ organismo que ocurre en un tiempo $T$ con tal y cual evento fisicoquímico del organismo que ocurre en $T$; ast, lo que es tener una creencia o sentir un dolor no dependerá de un tipo de estado fisicoquímico, sino que nuestros criterios normales de atribución de una creencia (o de cualquier otro estado mental) a un organismo decidirán si éste tiene creencias (u otros estados mentales) y, posteriormente, la ciencia nos dirá qué tipo de evento fisicoquímico se llevó a cabo en el momento en que el organismo tuvo, por ejemplo, tal y cual creencia particular.

5. El argumento de Armstrong a favor de la prioridad de la autoridad cientifica para decidir sobre lo que es el caso no parece claro:

a) El tipo de fisicalismo que Armstrong desea sostener es tanto epistémico - la presencia de estados mentales se explica en términos puramente fisicoquímicos- como metafísico: los estados mentales son, de hecho, estados fisicoquímicos del cuerpo. Pero epuede la ciencia decidir sobre lo que es el caso metafísicamente, o es una disciplina puramente epistémica (como explicamos y conocemos las cosas los hombres)?

¿Es verdad que sólo como un resultado de investigaciones cientificas se logra un consenso intelectual? No me parece que la respuesta sea evidentemente 'si'; pero suponiendo que sí, ¿sobre qué clase de cuestiones logra un consenso la ciencia? ¿Puede la evidencia científica resolver cuestiones de disputa en moral, estética, metafísica, religión...?

Aun si la ciencia estableciese que la única causa de $\mathrm{X}$ tipo de conducta en el hombre son los estados fisicoqufmicos tales y cuales del sistema nervioso

15 Cf. H. Putnam, "The mental life of some machines", Language, Mind and Reality, cap. 20, Cambridge University Press, 1970. 
central, este hecho parecería irrelevante para el problema filosófico que estamos tratando. El que toda la evidencia científica indique que la única causa de toda nuestra conducta es el funcionamiento del sistema nervioso central no ofrece garantía de que los estados mentales sean estados del sistema nervioso central; después de todo, puede argumentarse que ésta es la única causa fisica y que la ciencia sólo busca ese tipo de causas. Un cartesiano aceptaría toda la evidencia científica y diría que, efectivamente, los estados fisicoquímicos del sistema nervioso central son la única causa física de tal y cual conducta; pero que la causa de esa causa física es la "mente" del hombre. Que la ciencia estudia el cuerpo y la composición física del hombre y que este campo es absolutamente competente; pero que cuando hablo de mi sensación de dolor me refiero a dos cosas donde la ciencia no es competente - a saber, el "yo" y un estado de ese "yo"-, no al cuerpo del "yo".

b) Armstrong dice que toda la evidencia científica parece indicar que la naturaleza del hombre no es más que fisicoquímica; que podemos dar una explicación completa del hombre en términos puramente fisicoquímicos y que, por tanto, debemos tratar de elaborar una explicación de la naturaleza de la mente que sea compatible con esta evidencia.

Como Armstrong ve las cosas, el problema con los estados mentales es conceptual; pero el que la naturaleza de los estados sea ff́sica o "espiritual" parece no introducir ninguna diferencia filosófica sobre estos problemas: ellos son independientes de las investigaciones neurofisiológicas del cuerpo.

Lo primariamente importante, con respecto a los estados mentales, es tratar de explicar cómo es que obtenemos el concepto de estado mental, qué es lo que intuitivamente engloban nuestros conceptos mentales, qué es lo esencial y característico de un estado mental, qué significado tienen los enunciados sobre estados mentales, si refieren o no, cuál es su papel dentro del lenguaje, cómo y por qué podemos predicar la ocurrencia de estados mentales en Fulano, qué relación guardan nuestros conceptos mentales con la conducta manifiesta... Y todo esto antes de proponer cualquier posible identidad con ellos; porque, antes de tratar de identificar a los estados mentales con algo, necesitamos tener una correcta caracterización de lo que entendemos por un estado mental, a saber, a qué, cómo y cuándo aplicamos conceptos mentales, etcétera; de lo contrario estaremos identificando con estados físicos (en el caso de la tesis de la identidad) otras cosas y no lo que ordinariamente llamados 'estado mental'.

El problema es que si estos puntos no han sido bien aclarados - lo cual Armstrong está muy lejos de haber hecho- la pregunta ¿los estados mentales son estados físicos o tienen una naturaleza distinta a la f́sica? no tiene sentido; no podemos darle una respuesta (no podemos postular una posible identidad) si no hemos resuelto los problemas filosóficos que los estados mentales encierran. La pregunta necesita plantearse dentro de un contexto específico con bases teóricas que recojan todo lo que intuitivamente engloban nuestros conceptos mentales, que explique por qué es un error adjudicarles "propie- 
dades" $u$ otras cosas que no se encuentran necesariamente vinculadas a ellos, a qué, cómo y cuándo los aplicamos, para que sea posible buscarle una respuesta.

InSTITUto dE INVESTTGaciones FiLosóficas

Silvia Bello G.

Universidad Nacional Autónoma de México 